\title{
GLAD!
}

Revue sur le langage, le genre, les sexualités

$02 \mid 2017$

Varia

\section{Les dictionnaires collaboratifs en tant qu'objets discursifs, linguistiques et sociaux}

Résumé de thèse

Collaborative Dictionaries as Linguistic, Discursive and Social Objects

\section{Kaja Dolar}

\section{OpenEdition}

\section{Journals}

Édition électronique

URL : http://journals.openedition.org/glad/475

DOI : $10.4000 /$ glad. 475

ISSN : 2551-0819

Éditeur

Association GSL

\section{Référence électronique}

Kaja Dolar, "Les dictionnaires collaboratifs en tant qu'objets discursifs, linguistiques et sociaux », GLAD!

[En ligne], 02 | 2017, mis en ligne le 01 juin 2017, consulté le 21 janvier 2021. URL : http://

journals.openedition.org/glad/475; DOI : https://doi.org/10.4000/glad.475

Ce document a été généré automatiquement le 21 janvier 2021.

\section{$\circledast \oplus \Theta \Theta$}

La revue GLAD! est mise à disposition selon les termes de la Licence Creative Commons Attribution -

Pas d'Utilisation Commerciale - Pas de Modification 4.0 International. 


\section{Les dictionnaires collaboratifs en tant qu'objets discursifs, linguistiques et sociaux}

Résumé de thèse

Collaborative Dictionaries as Linguistic, Discursive and Social Objects

Kaja Dolar

\section{RÉFÉRENCE}

K. Dolar. 2017. Les dictionnaires collaboratifs en tant qu'objets discursifs, linguistiques et sociaux. Thèse de doctorat en Sciences du langage, sous la direction de Jean-François Jeandillou, Université Paris Ouest Nanterre La Défense

1 Dans le cadre de nos travaux, nous définissons la lexicographie collaborative - en nous appuyant sur Cotter et Damaso (2007), Meyer et Gurevych (2012), Granger (2012), etc. comme un genre dictionnairique qui intègre les contributions d'une communauté et qui crée, via l'internet, un espace virtuel où les contributeurs se soutiennent, collaborent et coopèrent dans la rédaction. Nous nous focalisons sur les dictionnaires collaboratifs généraux monolingues, un terrain qui semble très peu exploité actuellement. Nous nous concentrons en particulier sur les dictionnaires français La Parlure, Le dictionnaire collaboratif $d u$ français parlé et le dictionnaire slovène Razvezani jezik, Slovar žive slovenščine (que nous avons traduit La Langue déliée, Dictionnaire libre du slovène vivant).

2 Nous proposons une analyse en trois parties. Dans la première partie, nous décrivons les dictionnaires collaboratifs $\mathrm{du}$ point de vue lexicographique (typologie, macrostructure et accès aux entrées, microstructure et divers éléments des articles dictionnairiques), énonciatif (dispositif communicationnel, médium, caractère dialogique et polyphonique) et sociolinguistique (la question des contributeurs). Nous 
explorons également la question de la néologie et de la norme ainsi que les moyens de régulation du contenu.

3 La deuxième partie est consacrée à quatre phénomènes linguistiques, qui s'inscrivent de façon particulièrement intéressante dans le contexte de la lexicographie collaborative: le métalangage, le nom propre, le figement et la métaphore. En comparant ces phénomènes dans le contexte de la lexicographie traditionnelle, nous mettons en avant leurs différences et leurs spécificités dans le contexte des dictionnaires collaboratifs (par exemple le métalangage ordinaire/spontané, les dérivations et locutions à base de nom propre, la variation géographique des expressions figées, les métaphores créatives et les divers transferts sémantiques, etc.).

Dans la troisième partie, nous étudions la place du genre (gender) dans les dictionnaires collaboratifs, à travers les différentes désignations relevant du genre et de la sexualité (organes, actes, pratiques, orientation, etc.). En prolongement des thématiques traitées auparavant, nous analysons les expressions métaphoriques relevant du genre et de la sexualité. Nous examinons les métaphores désignant un caractère et un aspect physique masculin ou féminin d'une part ; puis nous étudions les métaphores relevant de la sexualité d'autre part. L'analyse montre une certaine dissymétrie: si les expressions métaphoriques désignant le sexe masculin et la poitrine féminine sont nombreuses, les matrices du sexe féminin et de l'orientation sexuelle se prêtent plutôt à d'autres types d'expressions, notamment la métonymie et la synecdoque. Même si le corpus présente un grand nombre de métaphores créatives qui apportent une innovation linguistique, elles restent néanmoins, au niveau du contenu, le plus souvent enracinées dans des schémas existants. Enfin, nous avons élargi notre étude à l'ensemble des désignations relevant du genre et de la sexualité, en ne nous limitant pas aux métaphores. Une approche quantitative nous a permis de dégager quelques tendances intéressantes : le nombre d'entrées décrivant les femmes est bien plus élevé que celui qui décrit les hommes. Elles se rapportent, le plus souvent, à l'aspect physique féminin. La catégorie des expressions se rapportant à la sexualité est extrêmement hétérogène et diversifiée. Le corpus met en avant des schémas traditionnels (par exemple, le plaisir relève du domaine masculin, la prostitution et le caractère libertin du féminin) ainsi que la matrice hétérosexuelle et l'hétéronormativité. Pourtant, certaines entrées subversives témoignent de changements, même si elles sont, pour l'instant, peu représentées.

5 Les trois axes explorés nous mènent à la conclusion que nous sommes confrontés à un objet novateur, un nouveau phénomène dans l'histoire de la lexicographie qui n'a pas encore été exploré et qui ouvre de nombreuses pistes prometteuses. 
INDEX

Thèmes : Actualités

Keywords : collaborative dictionary, collaborative lexicography, discursive linguistics, gender and language, enunciation, sociolinguistics, neologisms, norm, meta-language, proper name, set expressions, metaphor, gender, sexuality

Mots-clés : dictionnaire collaboratif, lexicographie collaborative, linguistique discursive, énonciation, sociolinguistique, néologisme, norme, métalangage, nom propre, figement, métaphore, genre, sexualité

\section{AUTEURS}

\section{KAJA DOLAR}

Docteure en sciences du langage. MoDyCo, Université Paris Ouest Nanterre La Défense Kaja Dolar est docteure en Sciences du langage. Ses recherches portent sur la lexicographie collaborative et s'inscrivent dans le cadre de la linguistique discursive, de la sémantique lexicale et de la lexicographie. 